\title{
Clinical Study \\ Resection and Immunotherapy for Recurrent Grade III Glioma
}

\author{
Iris Elens, ${ }^{1}$ Steven De Vleeschouwer, ${ }^{2}$ Femke Pauwels, ${ }^{3}$ and Stefaan Van Gool ${ }^{1,3,4}$ \\ ${ }^{1}$ Department of Child \& Women, University Hospital Leuven, Catholic University of Leuven, 3000 Leuven, Belgium \\ ${ }^{2}$ Department of Neurosurgery, University Hospital Leuven, Catholic University of Leuven, 3000 Leuven, Belgium \\ ${ }^{3}$ Department of Experimental Medicine, University Hospital Leuven, Catholic University of Leuven, 3000 Leuven, Belgium \\ ${ }^{4}$ Pediatric Hemato-Oncology, University Hospital Leuven, Herestraat 49, 3000 Leuven, Belgium
}

Correspondence should be addressed to Stefaan Van Gool, stefaan.vangool@med.kuleuven.be

Received 27 September 2011; Accepted 10 November 2011

Academic Editors: A. Rebollo and S. Vuckovic

Copyright () 2012 Iris Elens et al. This is an open access article distributed under the Creative Commons Attribution License, which permits unrestricted use, distribution, and reproduction in any medium, provided the original work is properly cited.

\begin{abstract}
Background. Despite surgery, radiotherapy, and chemotherapy, the prognosis of relapsed grade III gliomas remains poor. After promising results of immunotherapy in grade IV gliomas, we investigated its safety and efficacy in recurrent grade III gliomas. Methods. Thirty-nine patients received vaccines containing dendritic cells loaded with autologous tumor lysate after tumor resection. Progression-free survival (PFS) and overall survival (OS) were compared with those obtained after temozolomide (TMZ) treatment as found in the literature. Results. Median PFS and OS were 4.6 and 20.5, 3.4 and 18.8, 7.8 and 13.3 months in recurrent grade III astrocytoma, oligodendroglioma, and oligoastrocytoma, respectively. Compared with TMZ, no grade III/IV toxicity was reported and median OS tended to be higher although there was no difference in median PFS. The perceived benefit of immunotherapy was more pronounced in astrocytic tumors. Conclusions. We provide the first description of immunotherapy in recurrent grade III glioma as safe, promising, and feasible.
\end{abstract}

\section{Introduction}

Gliomas are primary brain tumors derived from the neuroepithelial tissue. According to their biological behavior and pathological appearance, the World Health Organization classified gliomas into four grades [1,2]. Grade III (anaplastic gliomas) among the gliomas with the worst prognosis. Gliomas were further subdivided according to the morphological resemblance they exhibit with normal lineages of glial cells, although evidence is lacking on whether the tumors really originate from these supposed precursors [3]. Our study focused on anaplastic astrocytoma (AA), anaplastic oligoastrocytoma (AOA), and anaplastic oligodendroglioma (AOD).

The current medical approach for these gliomas relies on a multimodal therapy that consists of maximal safe surgery to obtain symptomatic relief and collect brain tissue necessary for pathological diagnosis, followed by radiotherapy and/or chemotherapy to induce local control [4-7]. Frequent relapses have proven this strategy alone to be insufficient, prompting the elaboration of new treatments to improve the poor prognosis of these grade III gliomas. In the case of brain tumors, every strategy has to overcome the blood-brain barrier (BBB), which makes designing novel therapies even more challenging. Once relapse occurs in grade III gliomas, survival is usually short. Some patients will undergo a reresection before receiving chemotherapy [8-14]. For that, the most frequently used molecule nowadays is temozolomide (TMZ), an alkylating chemotherapeutic substance that is also administered to newly diagnosed grade IV/glioblastoma multiforme (GBM) patients. PCV (procarbazine, lomustine, vincristine) chemotherapy, or lomustine monotherapy, may be other options. Evidence-based guidelines concerning the approach of relapsed grade III gliomas are lacking and studies reporting survival rates frequently involve low numbers of patients.

Stimulating the patient's immune system to help eradicate a tumor is an approach that has been envisaged for long. There are several ways of stimulating the immune system, vaccination with mature autologous dendritic cells (DCs) being one of them. This treatment modality has already proven its safety and benefit in preclinical models [15-17] and in grade IV gliomas both in adults and children [18-23]. Immunotherapy also has been successfully applied in other tumors than tumors of the central nervous system [24-28]. The principle of this immunotherapeutic treatment consists 
in loading patient's DCs with autologous tumor lysate in vitro and injecting these loaded mature DCs into the patient. The aim is to activate specific cytotoxic $\mathrm{T}$ cells that will cross the broken BBB at the tumor site where they evoke local and specific antitumor activity.

This retrospective study focuses on the survival benefit of immunotherapy in patients with relapsed grade III gliomas included in the HGG-IMMUNO-2003 study. We aimed to compare progression-free survival (PFS), overall survival (OS), and toxicity with current data from studies in which TMZ was used. In addition, individual survival data of recurrent $\mathrm{AA}$ and $\mathrm{AOD}$ were analyzed in order to detect potential glioma subtype-specific efficiency.

\section{Materials and Methods}

2.1. Patients. Patients presenting between 2002 and 2010 with first or subsequent recurrent grade III glioma were included in this study. Relapse was defined as an increase of the tumor mass and/or augmented contrast enhancement on magnetic resonance imaging (MRI), with or without neurological symptoms. Once recurrence was confirmed, all patients underwent maximal safe surgery. None of our patients received chemotherapy thereafter. Patients were included if (1) a first or subsequent recurrent grade III glioma was documented, (2) they were between 3 and 70 years of age, (3) Karnofsky performance scale (KPS) equaled or exceeded 70 before the operation, (4) resection resulted in absent or minimal tumor volume $\left(<2 \mathrm{~cm}^{3}\right)$ and enough sterile and dry tumor tissue could be obtained to produce the vaccine, and (5) corticosteroids could be aborted shortly after the operation. Exclusion criteria were mentioned earlier [20].

2.2. Assessment of Extent of Tumor Resection before Vaccination. Total resection required both perioperative confirmation of the neurosurgeon and absence of any residual tumor mass on MRI or computed tomography scan without contrast performed within $72 \mathrm{~h}$ after the intervention. All other situations were classified as subtotal.

2.3. Tumor Lysate. After resection, tumor tissue was immediately transported to the laboratory where it was snapfrozen into liquid $\mathrm{N}_{2}$ without additives. To prepare tumor lysate, thawed tissue was homogenized mechanically in $\mathrm{NaCl}$ $0.9 \%$ containing $1 \%$ human serum albumin (HSA). After six freeze-thaw cycles, the homogenate was filtered through a Falcon filter $(0.70 \mu \mathrm{m})$ to obtain a lysate. Cell death was ascertained by tryptan blue exclusion assay and protein concentration in the lysate was measured by using the Coomassie blue staining method [29]. Finally, the lysate was irradiated (60 Gray) and kept frozen until use.

2.4. Preparation of Autologous DC. Peripheral blood mononuclear cells (PBMCs) were isolated from leukapheresis samples taken during the week following cessation of corticosteroids. Isolated PBMCs were kept frozen until use. Immature monocyte-derived DCs were generated out of monocytes which were obtained by adherence and loaded with autologous tumor lysate according to the procedure previously described [30-32]. At the time of loading, rTNF- $\alpha$ (Strathmann Biotec, Dengelsberg, Germany), rIL1- $\beta$ (Strathmann Biotec), and $\mathrm{PGE}_{2}$ (Prostin; Pfizer, Brussels, Belgium) were added to a concentration of 120,120 , and $20 \mu \mathrm{g} / \mathrm{mL}$, respectively. After $24 \mathrm{~h}$ of maturation, loaded DCs were resuspended in phosphate-buffered saline (PBS) containing $0.5 \%$ HSA. The quality of the DCs was assessed by the expression of HLA-DR, CD80, CD86, and CD83 as published earlier $[20,21,30,31]$.

2.5. Vaccination. Vaccines were administrated intradermally in the upper arms. After the injection, patients were kept in the hospital for observation during 30 minutes. There were four vaccination cohorts with slight modifications as described previously [23]. One, five, four, and twenty-nine patients were treated according to cohort A, B, C, and D, respectively. In the latter two cohorts-representing $85 \%$ of the patients - four vaccines were given, each at a one week interval. Thereafter, patients received booster injections consisting only of tumor autologous lysate. The number of booster injections depended on the quantity of tumor tissue excised at the time of the operation. In cohort D, imiquimod (Aldara) was applied at the injection site prior to vaccination because the maturation of the DCs in this cohort was performed without $\mathrm{PGE}_{2}$ [23].

2.6. Patient Assessment. All patients underwent clinical examination and analysis by imaging. A first MRI scan was performed 12 weeks postoperatively and then every three months or earlier if patients exhibited symptoms suggesting tumor progression.

2.7. Search Strategy. We consulted the Medline and Cochrane databases to find studies in which patients with recurrent grade III glioma were treated with TMZ and who could be used as control for our patients treated by immunotherapy. Different combinations of the following terms were used for the search: "anaplastic glioma, anaplastic astrocytoma, anaplastic oligodendroglioma, recurrence, TMZ, Temozolomide, survival" and we focused on prospective studies performed after 2000. We also included related articles. Relevant publications were critically appraised (http://www.cebm .net/). For this study, we only compared survival data with the data published on survival after TMZ treatment. We did not focus in particular to the treatment with PCV for recurrent $A O D$.

2.8. Statistical Analysis. Statistics were calculated with Prism software (version 5.02; Graphpad Software Inc., San Diego, CA, USA). Results were represented as Kaplan-Meier survival curves and analyzed using the LogRank test.

\section{Results}

3.1. Patient Data. Thirty-nine patients (median age $=43$ years, ranging from 7 to 69 with 6 patients below 18 years) 
TABLe 1: Patient characteristics.

\begin{tabular}{lccc}
\hline & AA & AOD & AOA \\
\hline$n$ patients & 18 & 13 & 8 \\
Male/female (\%) & $10(55) / 8(45)$ & $9(69) / 4(31)$ & $7(88) / 1(12)$ \\
Registration period & $2004-2010$ & $2004-2009$ & $2002-2010$ \\
Median age (range) & $30(7-62)$ & $42(38-69)$ & $32(11-56)$ \\
Median $n$ events* & 2 & $5(38) / 8(62)$ & 2 \\
1 event/>1 event (\%) & $9(50) / 9(50)$ & $3(23) / 10(77)$ & $5(63) / 3(37)$ \\
Total/subtotal resection (\%) & $11(61) / 7(39)$ & $8(62) / 5(38)$ & $3(37) / 5(63)$ \\
Initial grade III/dedifferentiation $(\%)$ & $17(94) / 1(6)$ & $7(53)$ & $2(25)$ \\
Patients alive $(\%)$ & $7(38)$ & & $3(63)$ \\
\hline
\end{tabular}

AA: anaplastic astrocytoma; AOD: anaplastic oligodendroglioma; AOA: anaplastic oligoastrocytoma.

$*$ : number of events prior to surgery and immunotherapy.

were included in the study. Patient data are summarized in Table 1. However, 50, 38, and $63 \%$ of patients with $\mathrm{AA}, \mathrm{AOD}$ and $\mathrm{AOA}$, respectively, presented with a first episode of relapse. The other patients had been subjected to multiple relapses prior to neurosurgery and subsequent immunotherapy. The highest number of total resections was achieved in AA (61\%), while this was achieved in only $23 \%$ and $37 \%$ of patients with AOD and AOA, respectively. In $94 \%$ of the patients with $\mathrm{AA}$, the first event was a grade III astrocytoma. This was, respectively, 62 and $37 \%$ in patients with AOD and AOA, showing a larger proportion of grade II tumors at time of the first event with subsequent dedifferentiation for these two tumor entities.

A median number of 8 (range 4-16), 8 (range 4-14), and 7 (range 4-12) vaccines were administered to the patients presenting with $\mathrm{AA}, \mathrm{AOD}$, and $\mathrm{AOA}$, respectively. A median number of $5 \times 10^{6}$ (range $\left.0.88-23.3 \times 10^{6}\right)$ DCs was injected per vaccination.

3.2. Clinical Outcome. Survival data of AA, AOD, and AOA are represented in Table 2. Median PFS and OS were, respectively, 4.6 and 20.5 months for patients with AA, 3.4 and 18.8 months for patients with AOD, and 7.8 and 13.3 months for patients with AOA. At time of analysis, the survival rate was 38,53 and $25 \%$ in patients who presented with $\mathrm{AA}, \mathrm{AOD}$, and AOA, respectively, with a median (range) followup of 19.5 (2.7-51.6), 14.8 (5.8-29.9), and 11 (4.620.3) months.

The on-line search yielded seven studies that used TMZ treatment $[8-14]$. They are described in detail in Table 3. The results of our study obtained with immunotherapy after reresection was included into the table. No data on OS beyond 12-months with TMZ were available in the literature at the time of our investigation. Median OS of AA patients ranged between 12.3 and 14.6 after TMZ treatment. At twelve months, immunotherapy attained an OS of $76.5 \%$, while TMZ treatment resulted in a 12 month OS ranging from $56 \%$ to $59 \%$. OS after 24 and 48 months of immunotherapy remained stable.

For AOD, the median OS observed in our patients was similar to the range of 10-18 months obtained with TMZ. With immunotherapy, 12 month OS concerned $77 \%$ while it ranged from 46 to $71 \%$ in patients with TMZ. Nearly forty
TABLE 2: AA, AOD, and AOA characteristics.

\begin{tabular}{lccc}
\hline & AA & AOD & AOA \\
\hline Median medical history prior to & 39.5 & 118.4 & 72 \\
immunotherapy (m) & 20.5 & 18.8 & 13.3 \\
Median OS (m) & 4.6 & 3.4 & 7.8 \\
Median PFS (m) & 15.9 & 15.4 & 5.5 \\
Median interval PFS/death (m) & 37.5 & 33.3 & 66 \\
6 months PFS (\%) & 37.5 & 25 & 50 \\
12 months PFS (\%) & 30 & 25 & 25 \\
24 months PFS (\%) & 94.2 & 92.3 & 75 \\
6 months OS (\%) & 76.5 & 77 & 62.5 \\
12 months OS (\%) & 41.2 & 39.6 & 46.9 \\
\hline 24 months OS (\%) & &
\end{tabular}

OS: Overall survival, PFS: progression free survival, AA: anaplastic astrocytoma, AOD: anaplastic oligodendroglioma, AOA: anaplastic oligoastrocytoma.

percent of patients had an OS survival longer than 24 months when treated with immunotherapy at time of relapse.

In our study, the medical history of patients with AA was in median 78.9 months shorter than that of patients with AOD. While patients with AOD had a longer survival when measured from the moment of diagnosis, OS of AA and AOD after immunotherapy evolved similarly. Figure 1 illustrates the median time line of the medical history of AA and AOD patients.

Since total tumor resection was previously reported to correlate with longer survival $[4,20,23,33]$, we compared patient survival data as a function of complete and incomplete removal of the tumor. In our total population, patients lived significantly longer when the entire tumor mass was removed (Figure 2(a), LogRank test: $P$-value $=$ $0.01)$. Subgroup analysis revealed analogous observations for the different tumor subtypes (Figures 2(b)-2(d)) although no significance was achieved.

Dedifferentiation was defined as a grade III tumor on pathological examination of tumor tissue with previous histological diagnosis of a grade II glioma. In the case of dedifferentiated glioma grade IV (secondary GBM), earlier publications mentioned a better prognosis of dedifferentiated 


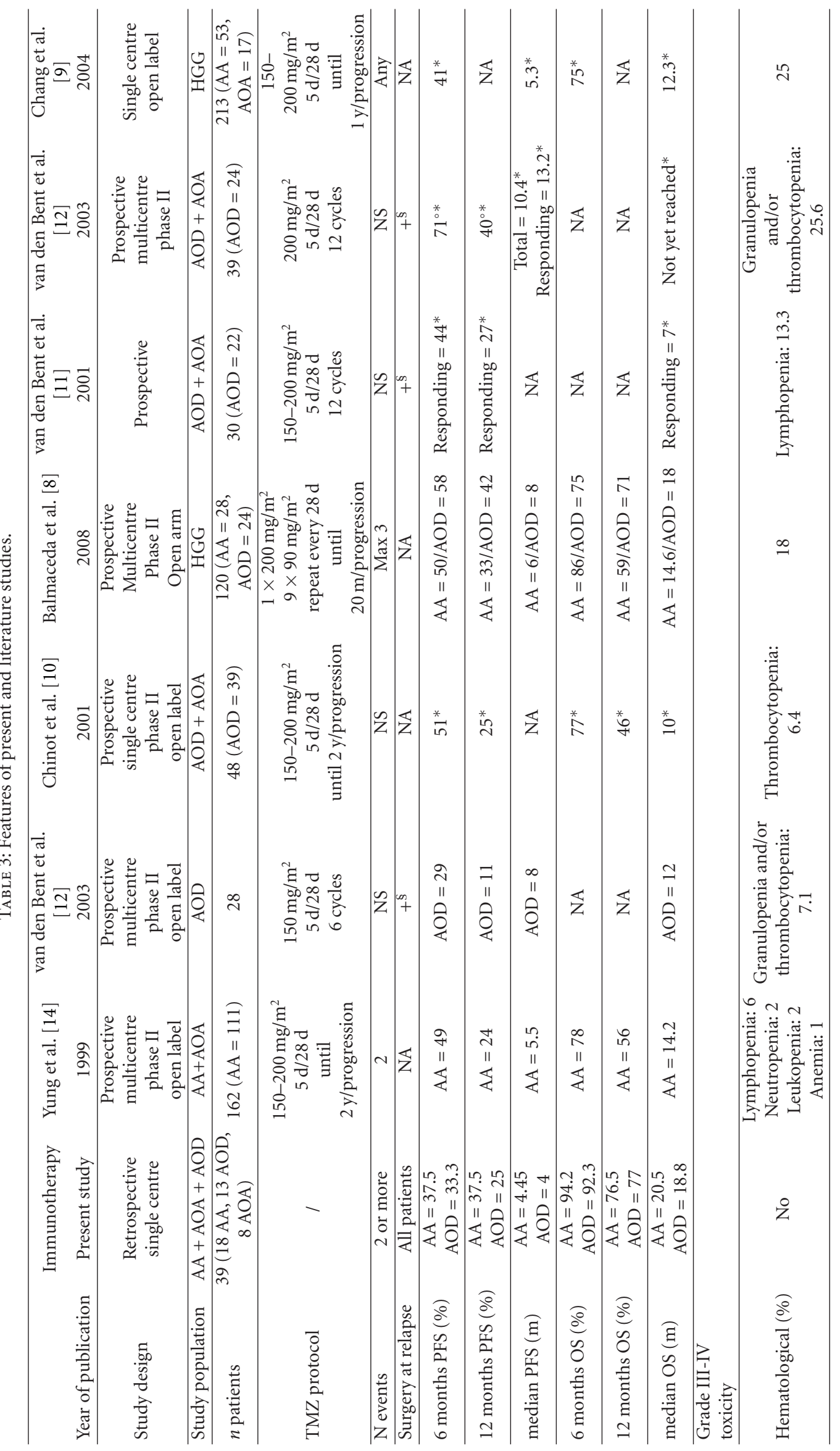




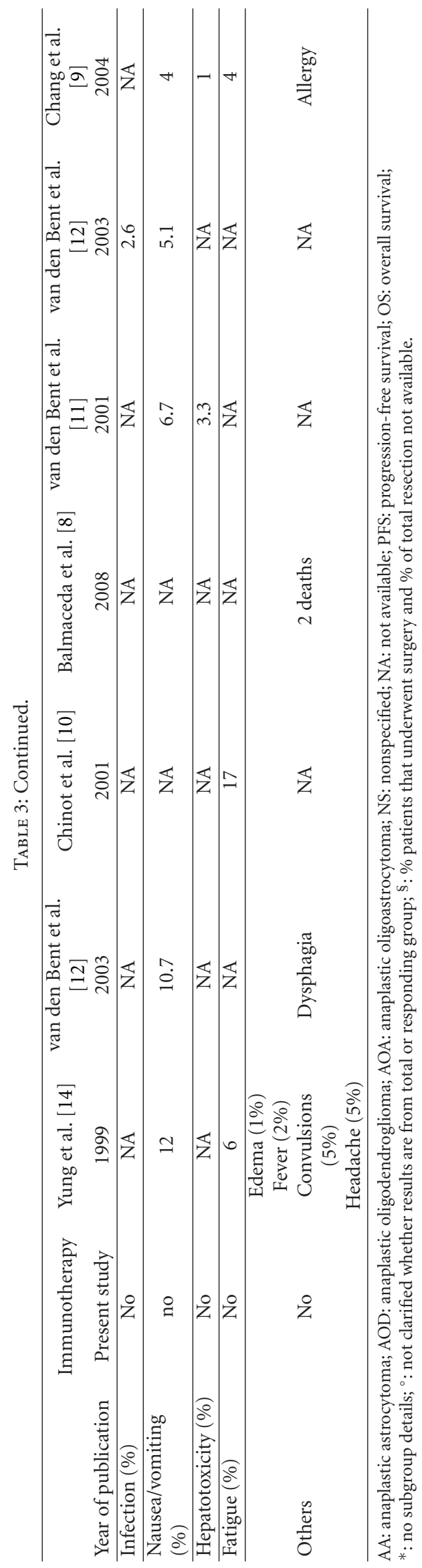




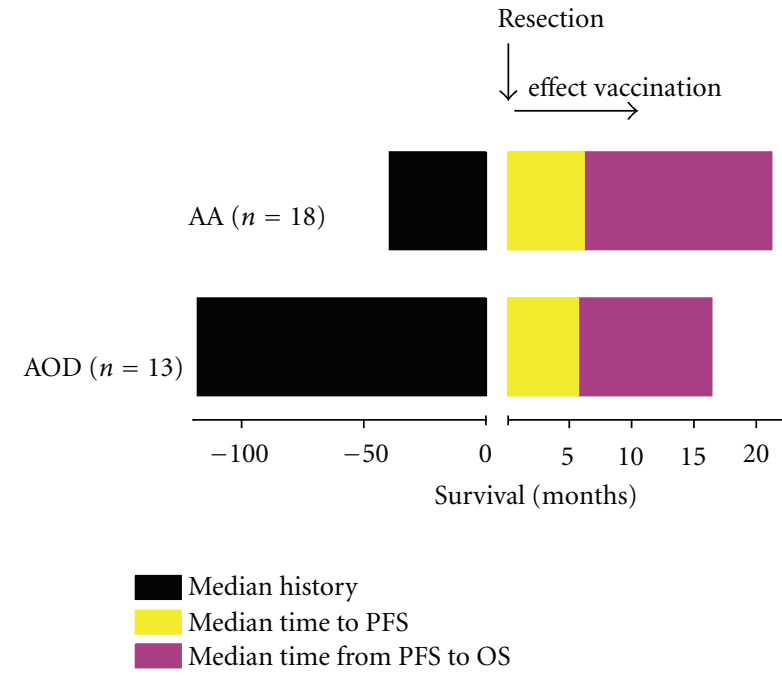

FIGURE 1: Column analysis presenting median medical history for $\mathrm{AA}$ and AOD before and after immunotherapy. The medical history of patients with AA was in median 78.9 months shorter than that of patients with AOD while the OS after immunotherapy evolves similar. AA: anaplastic astrocytoma, AOD: anaplastic oligodendroglioma.

tumors as compared with primary glioma [34-38]. We aimed to verify this observation also in our population of grade III tumor patients. At 24 months, we recorded a trend to better survival in patients in whom a dedifferentiated tumor was diagnosed (60.6\% versus $34.4 \%$ ). However, at 48 months, this difference was no longer apparent (17.3\% versus $20.2 \%)$.

3.3. Toxicity. We evaluated the toxicity of immunotherapy in our patient cohort according to the CTC criteria [39]. No cases of grade III-IV toxicity were observed. Side effects were headache, fatigue, fever, generalized itching, vomiting, and flu-like symptoms. No autoimmune symptoms were observed. All patients developed a local reaction at the site of vaccination. Compared with immunotherapy, TMZ elicited more severe toxicity reactions. Most frequently reported grade III-IV toxicity after TMZ included hematological symptoms, infections, nausea and vomiting, fatigue, hepatotoxicity, fever, convulsions, and headache (Table 3). In these cases, cessation of the TMZ therapy was rarely required but dose reduction was performed at the clinician's discretion.

\section{Discussion}

This study summarizes our experiences with immunotherapy in 39 patients presenting with recurrent grade III glioma. After resection of the recurrent tumor, the patients were vaccinated with autologous mature DC loaded with autologous tumor lysate. This type of immunotherapeutic treatment has already been successfully applied in the case of grade IV gliomas [20,21,30], but the present report is the first exclusively focusing on immunotherapy in a relatively large series of patients with relapsed grade III glioma's treated with immunotherapy. The treatment was well tolerated with no major toxicity issue. Moreover, a two-year tumor-free status and overall survival was obtained in a subgroup of $41.2,39.6$, and $46.9 \%$, representing 7,5 and 3 patients in AA, $\mathrm{AOD}$, and AOA, respectively.

Immunotherapy is not a stand-alone procedure but is adjuvant to tumor resection. Resection has two longterm objectives: it provides tumor tissue for preparing the lysate and it also reduces the tumor burden prior to immunotherapy. The latter point is crucial, as we have observed that the extent of resection has a prognostic effect on survival, which is in line with our earlier series of patients with grade IV relapsed glioma treated with surgery and subsequent immunotherapy $[7,19,20,23,33,40]$.

In addition to the extent of resection, we explored in our patient group some other potential risk factors that can be associated with the immunotherapeutic treatment. In contrast with previous reports, we did not observe any survival benefit related to age, $[20,23,40]$. Also, the more aggressive evolution of initial grade III versus dedifferentiated tumors, though observed at 24 months, was not seen after 48 months postvaccination. We finally compared survival data of patients with $\mathrm{AA}, \mathrm{AOD}$, and AOA. AOD is reported to evolve less aggressively than AA $[1,41]$, which was confirmed here by the obvious difference in medical history between AOD and AA. If immunotherapy had the same ability to induce tumor-free status for both tumor subtypes, we would expect a longer OS in AOD proportionally to the difference in medical history. However, we recorded opposite results. This leads us to hypothesize that astrocytic tumors are more responsive to immunotherapy than $\mathrm{AOD}$, even if the $\mathrm{OS}$ in the three patients with AOD in whom the tumor mass was completely removed is still $100 \%$ at 30 months, performing much better than any published data so far. The long PFS and short OS of patients with AOA was an unexpected observation but it may be biased by the low number of patients. The same applies to data on the risk factors. They should be interpreted with caution as our patient group is still too small to perform multivariate analysis and define independent risk factors.

In the frame of our evaluation of immunotherapy as a new therapeutic opportunity for grade III gliomas, we compared our data with data on TMZ treatment. Since our study was a single-arm study, we could only compare it with literature data. The first parameter that we compared was the toxicity of the two treatments. There were no cases of grade III to IV toxicity during and after immunotherapy. TMZ, on the contrary, induced well-known grade III to IV toxicities, as described in the reference group [8-14]. Although these sideeffects rarely forced to therapy cessation, they did have an important impact, not only on the necessary dose reductions but most of all on the quality of life of patients. This makes immunotherapy an attractive strategy when quality of life matters.

PCV chemotherapy has been applied in recurrent grade III oligodendroglioma as described earlier [42, 43]. Median PFS and OS were 10 and 20 months in the patient group described by van den Bent et al. [42]. Brandes et al. observed a median PFS and OS of 12.3 and 30.7 months in 37 patients with recurrent anaplastic oligodendroglioma [43]. 


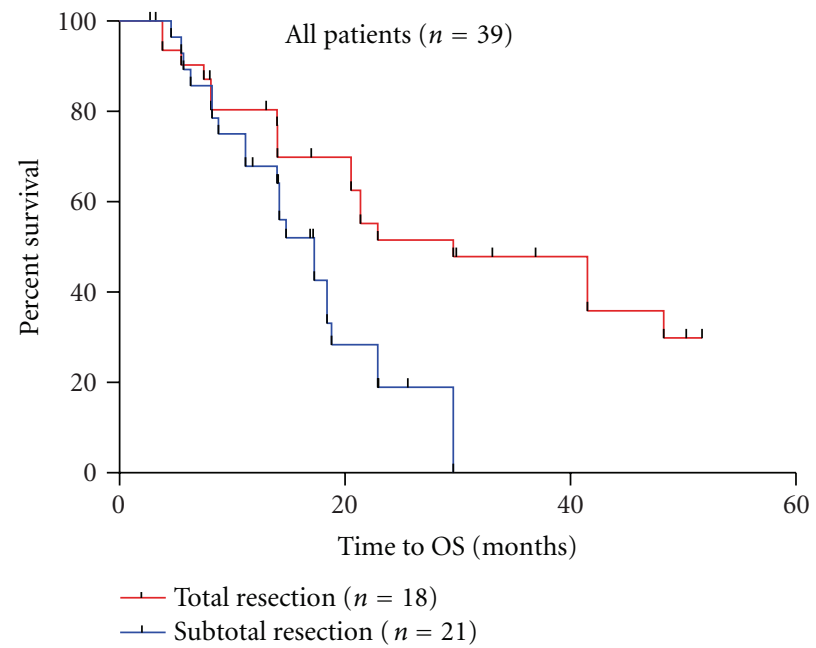

(a)

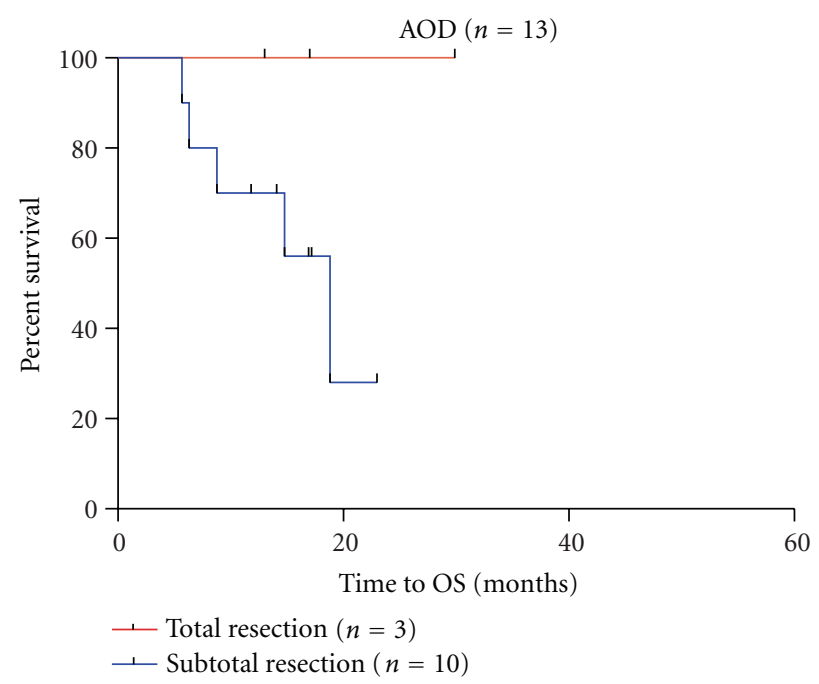

(c)

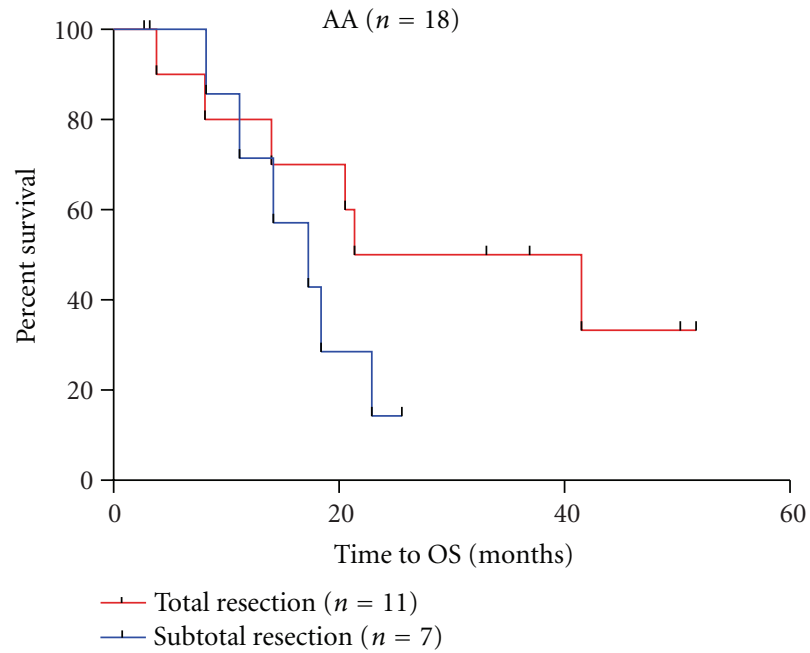

(b)

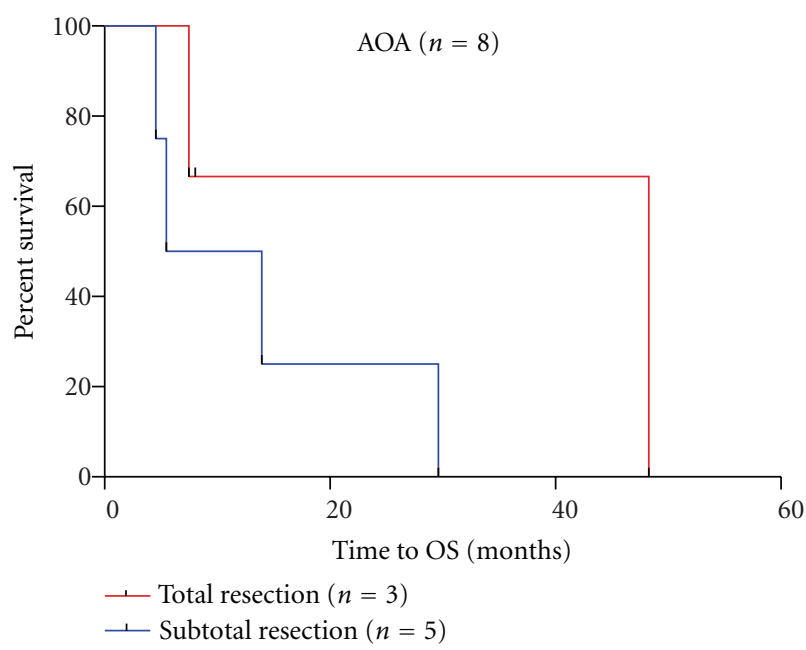

(d)

FIgURE 2: Kaplan-Meyer overall survival curves after immunotherapy following total or subtotal resection for all glioma grade III patients together (a), and for separate glioma subtypes; AA (b), AOD (c), and AOA (d). Survival curves were statistically compared with the LogRank test: in (a) $P=0.01$, in (b) $P=0.2$, in (c) $P=0.17$, and in (d) $P=0.22$. OS: overall survival, AA: anaplastic astrocytoma, AOD: anaplastic oligodendroglioma, AOA: anaplastic oligoastrocytoma.

Six months PFS, 12 months PFS, 6 months OS, and 12 months OS for these patients attained to $72 \%, 52 \%, 83 \%$, and $83 \%$, respectively.

Concerning survival data, our analysis showed a trend towards longer survival with immunotherapy as compared to TMZ, but inherent weaknesses of this observational study are apparent. The number of patients in both our patient and the reference group is low. In addition, although exact data are not reported, most patients in the reference group were presumably not reoperated at the time of recurrence. When analyzing data on recurrent malignancies, the medical history of the patients is very heterogeneous concerning number of events, previous surgical procedures, and radioand/or chemotherapy. Other points of diversity include the wide range in age and vaccination details. Based on empirical observations throughout the years, preparation and administration schedule of the injections were modified [15] (booster injections, application of imiquimod). Nevertheless, although there were some survival differences between early and later patient cohorts, they never reached significant levels [23]. Furthermore, a possible bias in our observations may have been introduced through the survival benefit of total versus subtotal resection, as we saw a significantly better survival in patients in whom total resection was performed. The higher number of total resections in the AA group could therefore play a role and interfere with the longer OS observed in this group. Monitoring of the immune response after DC vaccination has been tested out by our and other groups in previous publications [18]. However, the current available techniques failed to represent 
a reliable relationship between in vitro results and in vivo clinical antitumor response. Therefore, we opted for not applying immunomonitoring to evaluate the efficacy of DC vaccination. In spite of these weaknesses, our results advocate to consider immunotherapy as potential useful approach for reoperable, relapsed grade III gliomas.

Overall, the data presented in this study add knowledge to the growing experience about immunotherapy as a treatment for patients with malignant glioma at time of relapse. While most studies include patients with grade IV tumors, this study summarizes the results that we obtained on immunotherapy for patients with relapsed AA, AOD, and AOA. The treatment is feasible without major toxicity. The data on OS suggest that further work should be initiated on immunotherapy for recurrent grade III gliomas, particularly in those patients in whom a substantial resection can be performed.

\section{Abbreviations}

AA: Anapastic astrocytoma

AOA: Anaplastic oligoastrocytoma

AOD: Anaplastic oligodendroglioma

BBB: Blood-brain-barrier

DCs: Dendritic cells

GBM: Glioblastoma multiforme

OS: Overall survival

PBMCs: Peripheral blood mononuclear cells

PCV: Procarbazine, lomustine, vincristine

PFS: Progression free survival

TMZ: Temozolomide.

\section{Acknowledgments}

The authors would like to thank the members of the GMP laboratory facility: Goedele Stegen, Anaïs Van Hoylandt, Elke Nackers, Katja Vandenbrande, and Vallentina Schaiko. Fundings were provided by the Olivia Hendrickx Research Fund, the Herman Memorial Research Fund, the James E. Kearney Memorial Fund, FWO-V, IWT (TBM Program) and Stichting tegen Kanker. The authors are very grateful to the neuro-oncology team in the hospital for the fruitful patient discussion, and the staff of the Laboratory of Experimental Immunology for basic scientific discussions.

\section{References}

[1] H. Ohgaki and P. Kleihues, "Epidemiology and etiology of gliomas," Acta Neuropathologica, vol. 109, no. 1, pp. 93-108, 2005.

[2] D. N. Louis, H. Ohgaki, O. D. Wiestler et al., "The 2007 WHO classification of tumours of the central nervous system," Acta Neuropathologica, vol. 114, pp. 97-109, 2007.

[3] S. Pfister, C. Hartmann, and A. Korshunov, "Histology and molecular pathology of pediatric brain tumors," Journal of Child Neurology, vol. 24, no. 11, pp. 1375-1386, 2009.

[4] G. Dresemann, "Temozolomide in malignant glioma," Journal of OncoTargets and Therapy, vol. 3, pp. 139-146, 2010.

[5] M. K. Khan, G. K. Hunter, M. Vogelbaum, J. H. Suh, and S. T. Chao, "Evidence-based adjuvant therapy for gliomas: current concepts and newer developments," Indian Journal of Cancer, vol. 46, no. 2, pp. 96-107, 2009.

[6] R. A. Kristof, G. Neuloh, V. Hans et al., "Combined surgery, radiation, and PCV chemotherapy for astrocytomas compared to oligodendrogliomas and oligoastrocytomas WHO grade III," Journal of Neuro-Oncology, vol. 59, no. 3, pp. 231-237, 2002.

[7] R. P. Sanders, M. Kocak, P. C. Burger, T. E. Merchant, A. Gajjar, and A. Broniscer, "High-grade astrocytoma in very young children," Pediatric Blood and Cancer, vol. 49, no. 7, pp. 888-893, 2007.

[8] C. Balmaceda, D. Peereboom, S. Pannullo et al., "Multiinstitutional phase II study of temozolomide administered twice daily in the treatment of recurrent high-grade gliomas," Cancer, vol. 112, no. 5, pp. 1139-1146, 2008.

[9] S. M. Chang, P. Theodosopoulos, K. Lamborn et al., "Temozolomide in the treatment of recurrent malignant glioma," Cancer, vol. 100, no. 3, pp. 605-611, 2004.

[10] O. L. Chinot, S. Honore, H. Dufour et al., "Safety and efficacy of temozolomide in patients with recurrent anaplastic oligodendrogliomas after standard radiotherapy and chemotherapy," Journal of Clinical Oncology, vol. 19, no. 9, pp. 24492455, 2001.

[11] M. J. van den Bent, F. Keime-Guibert, A. A. Brandes et al., "Temozolomide chemotherapy in recurrent oligodendroglioma," Neurology, vol. 57, no. 2, pp. 340-342, 2001.

[12] M. J. van den Bent, O. Chinot, W. Boogerd et al., "Second-line chemotherapy with temozolomide in recurrent oligodendroglioma after PCV (procarbazine, lomustine and vincristine) chemotherapy: EORTC Brain Tumor Group phase II study 26972," Annals of Oncology, vol. 14, no. 4, pp. 599-602, 2003.

[13] M. J. van den Bent, M. J. Taphoorn, A. A. Brandes et al., "Phase II study of first-line chemotherapy with temozolomide in recurrent oligodendroglial tumors: the European Organization for Research and Treatment of Cancer Brain Tumor Group Study 26971," Journal of Clinical Oncology, vol. 21, no. 13, pp. 2525-2528, 2003.

[14] W. K. Yung, M. D. Prados, R. Yaya-Tur et al., "Multicenter phase II trial of temozolomide in patients with anaplastic astrocytoma or anaplastic oligoastrocytoma at first relapse. Temodal Brain Tumor Group," Journal of Clinical Oncology, vol. 17, no. 9, pp. 2762-2771, 1999.

[15] H. Ardon, B. Verbinnen, W. Maes, T. Beez, S. van Gool, and S. de Vleeschouwer, "Technical advancement in regulatory $\mathrm{T}$ cell isolation and characterization using CD127 expression in patients with malignant glioma treated with autologous dendritic cell vaccination," Journal of Immunological Methods, vol. 352, no. 1-2, pp. 169-173, 2010.

[16] W. Maes, C. Deroose, V. Reumers et al., "In vivo bioluminescence imaging in an experimental mouse model for dendritic cell based immunotherapy against malignant glioma," Journal of Neuro-Oncology, vol. 91, no. 2, pp. 127-139, 2009.

[17] W. Maes, G. G. Rosas, B. Verbinnen et al., "DC vaccination with anti-CD25 treatment leads to long-term immunity against experimental glioma," Neuro-Oncology, vol. 11, no. 5, pp. 529-542, 2009.

[18] H. Ardon, S. van Gool, I. S. Lopes et al., "Integration of autologous dendritic cell-based immunotherapy in the primary treatment for patients with newly diagnosed glioblastoma multiforme: a pilot study," Journal of Neuro-Oncology, vol. 99, no. 2, pp. 261-272, 2010.

[19] H. Ardon, S. de Vleeschouwer, F. van Calenbergh et al., "Adjuvant dendritic cell-based tumour vaccination for children with 
malignant brain tumours," Pediatric Blood and Cancer, vol. 54, no. 4, pp. 519-525, 2010.

[20] S. de Vleeschouwer, S. Fieuws, S. Rutkowski et al., "Postoperative adjuvant dendritic cell-based immunotherapy in patients with relapsed glioblastoma multiforme," Clinical Cancer Research, vol. 14, no. 10, pp. 3098-3104, 2008.

[21] S. de Vleeschouwer, M. Rapp, R. V. Sorg et al., "Dendritic cell vaccination in patients with malignant gliomas: current status and future directions," Neurosurgery, vol. 59, no. 5, pp. 988999, 2006.

[22] N. K. Foreman and A. M. Donson, "Tumor vaccination for malignant brain tumors," Pediatric Blood and Cancer, vol. 54, no. 4, pp. 495-496, 2010.

[23] S. van Gool, W. Maes, H. Ardon, T. Verschuere, S. van Cauter, and S. de Vleeschouwer, "Dendritic cell therapy of high-grade gliomas," Brain Pathology, vol. 19, no. 4, pp. 694-712, 2009.

[24] C. G. Drake, "Prostate cancer as a model for tumour immunotherapy," Nature Reviews Immunology, vol. 10, no. 8, pp. 580593, 2010.

[25] S. van Gool, "The dendritic therapy with its potential applications in pancreatic cancer," Acta Gastro-Enterologica Belgica, vol. 72, no. 3, pp. 338-343, 2009.

[26] H. van Poppel, S. Joniau, and S. W. van Gool, "Vaccine therapy in patients with renal cell carcinoma," European Urology, vol. 55, no. 6, pp. 1333-1342, 2009.

[27] J. Weber, "Immunotherapy for melanoma," Current Opinion in Oncology, vol. 23, pp. 163-169, 2011.

[28] J. M. Timmerman and R. Levy, "Dendritic cell vaccines for cancer immunotherapy," Annual Review of Medicine, vol. 50, pp. 507-529, 1999.

[29] M. M. Bradford, "A rapid and sensitive method for the quantitation of microgram quantities of protein utilizing the principle of protein dye binding," Analytical Biochemistry, vol. 72, no. 1-2, pp. 248-254, 1976.

[30] S. de Vleeschouwer, M. Arredouani, M. Adé et al., "Uptake and presentation of malignant glioma tumor cell lysates by monocyte-derived dendritic cells," Cancer Immunology, Immunotherapy, vol. 54, no. 4, pp. 372-382, 2005.

[31] S. de Vleeschouwer, F. van Calenbergh, P. Demaerel et al., "Transient local response and persistent tumor control in a child with recurrent malignant glioma: treatment with combination therapy including dendritic cell therapy: case report," Journal of Neurosurgery, vol. 100, no. 5, pp. 492-497, 2004.

[32] S. Rutkowski, S. de Vleeschouwer, E. Kaempgen et al., "Surgery and adjuvant dendritic cell-based tumour vaccination for patients with relapsed malignant glioma, a feasibility study," British Journal of Cancer, vol. 91, no. 9, pp. 1656-1662, 2004.

[33] R. K. Hales, O. Shokek, P. C. Burger et al., "Prognostic factors in pediatric high-grade astrocytoma: the importance of accurate pathologic diagnosis," Journal of Neuro-Oncology, vol. 99, no. 1, pp. 65-71, 2010.

[34] H. Ohgaki and P. Kleihues, "Genetic alterations and signaling pathways in the evolution of gliomas," Cancer Science, vol. 100, no. 12, pp. 2235-2241, 2009.

[35] S. Nobusawa, T. Watanabe, P. Kleihues, and H. Ohgaki, "IDH1 mutations as molecular signature and predictive factor of secondary glioblastomas," Clinical Cancer Research, vol. 15, no. 19, pp. 6002-6007, 2009.

[36] H. Ohgaki, P. Dessen, B. Jourde et al., "Genetic pathways to glioblastoma: a population-based study," Cancer Research, vol. 64, no. 19, pp. 6892-6899, 2004.

[37] H. Yan, D. W. Parsons, G. Jin et al., "IDH1 and IDH2 mutations in gliomas," New England Journal of Medicine, vol. 360, no. 8, pp. 765-773, 2009.
[38] M. Eoli, F. Menghi, M. Bruzzone et al., "Methylation of O6-methylguanine DNA methytransferase and loss of heterozygosity on $19 \mathrm{q}$ and/or $17 \mathrm{p}$ are overlapping features of secondary glioblastomas with prolonged survival," Clinical Cancer Research, vol. 13, no. 9, pp. 2606-2613, 2007.

[39] "CTC criteria version 3.0. Cancer Therapy Evaluation Program, Common Terminology Criteria for Adverse Events version 3.0, DCTD, NCI, NIH, DHHS," 2006.

[40] C. Lebrun, D. Fontaine, A. Ramaioli et al., "Long-term outcome of oligodendrogliomas," Neurology, vol. 62, no. 10, pp. 1783-1787, 2004.

[41] H. Ohgaki and P. Kleihues, "Population-based studies on incidence, survival rates, and genetic alterations in astrocytic and oligodendroglial gliomas," Journal of Neuropathology and Experimental Neurology, vol. 64, no. 6, pp. 479-489, 2005.

[42] M. van den Bent, J. Kros, J. Heimans et al., "Response rate and prognostic factors of recurrent oligodendroglioma treated with procarbazine, CCNU, and vincristine chemotherapy. Dutch Neuro-oncology Group," Neurology, vol. 51, no. 4, pp. 1140-1145, 1998.

[43] A. Brandes, A. Tosoni, F. Vastola et al., "Efficacy and feasibility of standard procarbazine, lomustine, and vincristine chemotherapy in anaplastic oligodendroglioma and oligoastrocytoma recurrent radiotherapy. A phase II study," Cancer, vol. 101, no. 9, pp. 2079-2085, 2004. 


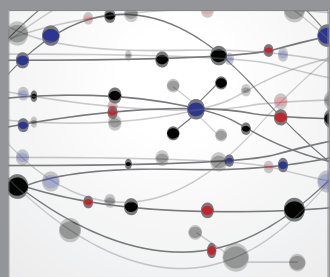

The Scientific World Journal
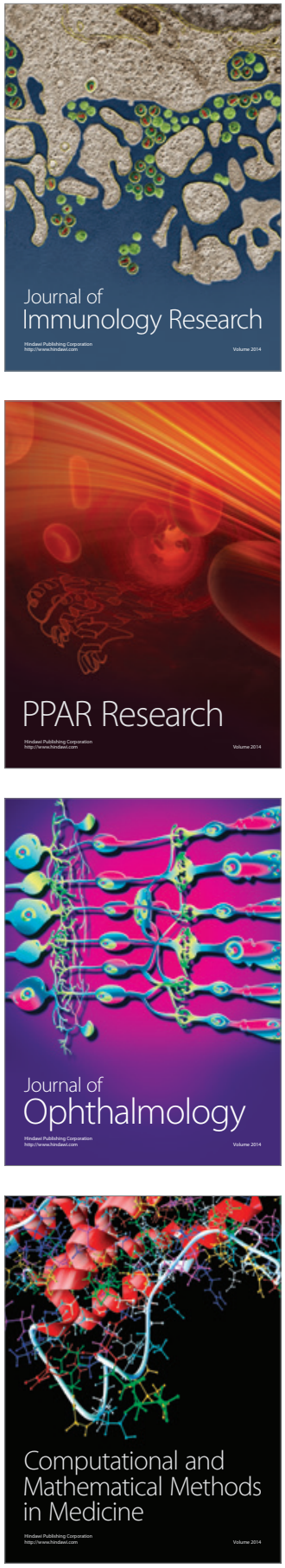

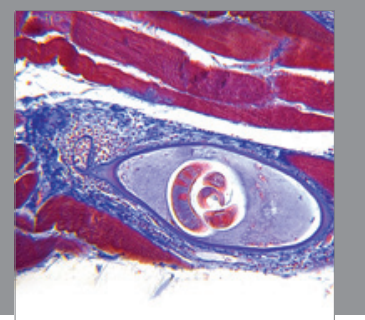

Gastroenterology

Research and Practice
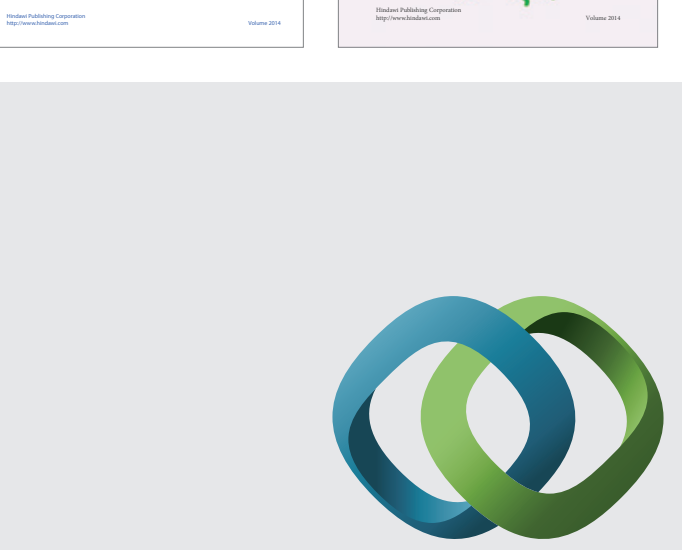

\section{Hindawi}

Submit your manuscripts at

http://www.hindawi.com
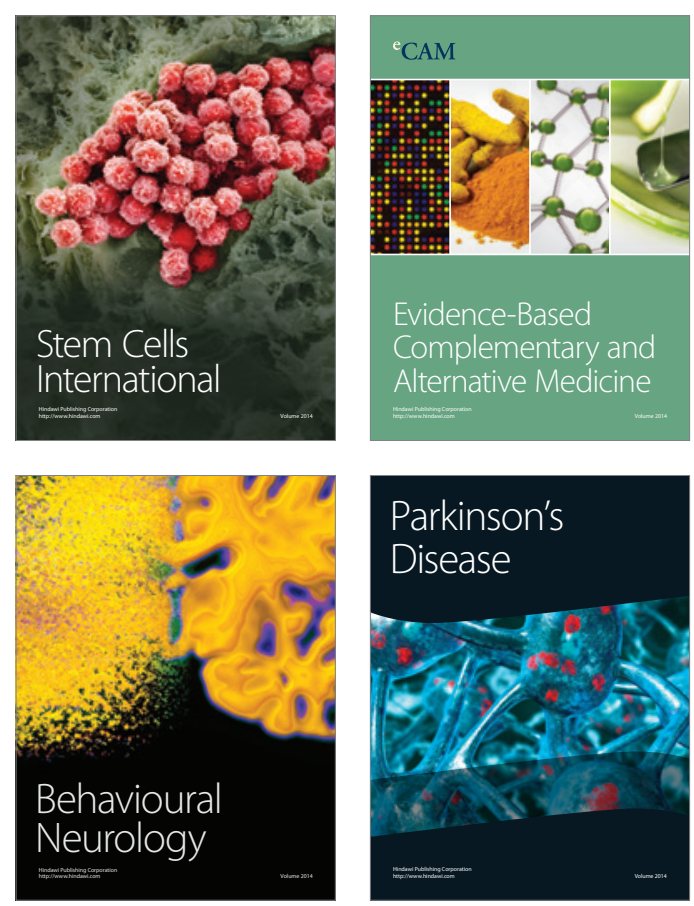

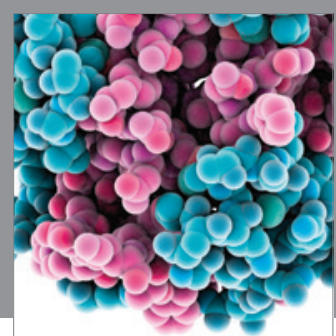

Journal of
Diabetes Research

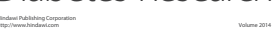

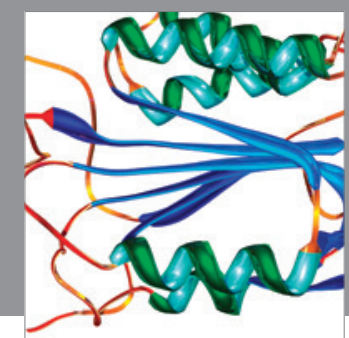

Disease Markers
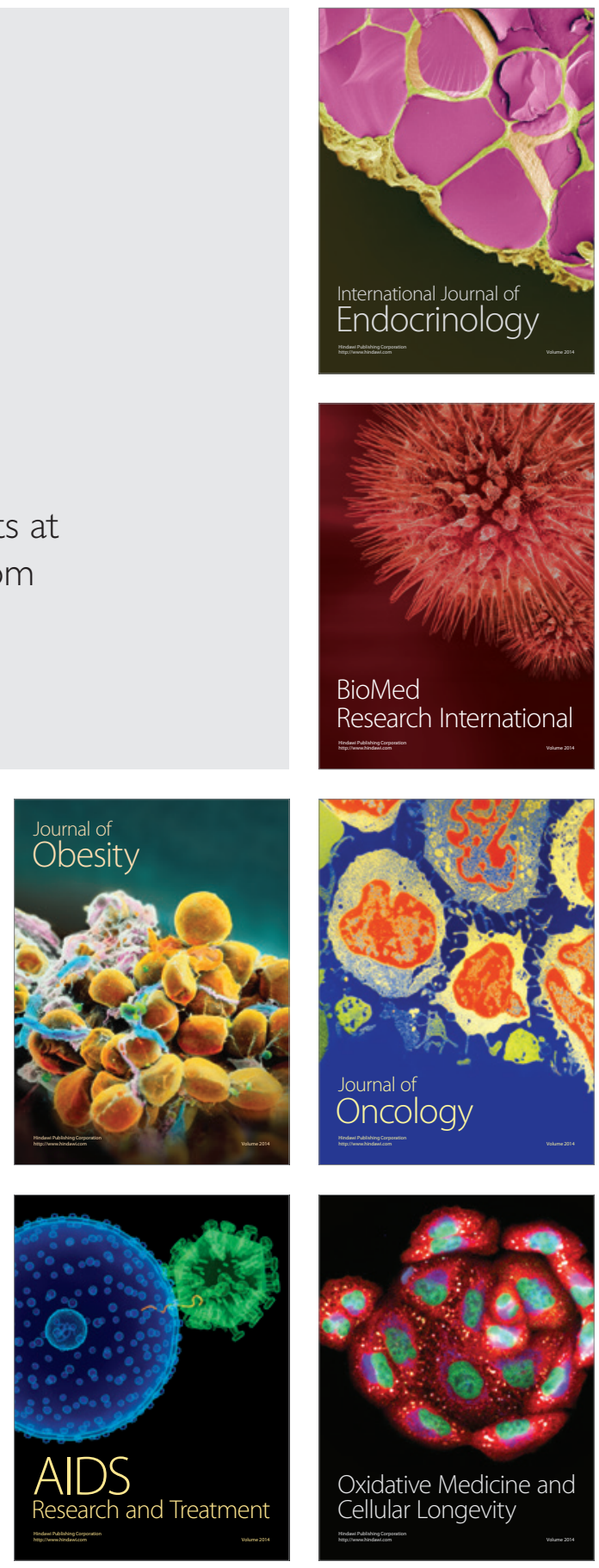